\title{
Asymptotic Harmonic Behavior in the Prime Number Distribution
}

\section{Maurice H. P. M. van Putten}

Sejong University, Seoul, South Korea

Email: $\underline{\text { mvp@sejong.ac.kr }}$

Received 16 June 2014; revised 19 July 2014; accepted 29 July 2014

Copyright (C) 2014 by author and Scientific Research Publishing Inc.

This work is licensed under the Creative Commons Attribution International License (CC BY).

http://creativecommons.org/licenses/by/4.0/

(c) (i) Open Access

\section{Abstract}

We consider $\Phi(x)=x^{-\frac{1}{4}}\left[1-2 \sqrt{x} \sum \mathrm{e}^{-p^{2} \pi x} \ln p\right]$ on $x>0$, where the sum is over all primes $p$. If $\Phi$ is bounded on $x>0$, then the Riemann hypothesis is true or there are infinitely many zeros $\operatorname{Rez}_{k}>\frac{1}{2}$. The first 21 zeros give rise to asymptotic harmonic behavior in $\Phi(x)$ defined by the prime numbers up to one trillion.

\section{Keywords}

Prime Number Distribution, Summation, Regularization

\section{Introduction}

The Riemann-zeta function is the analytic extension of

$$
\zeta(z)=1+\frac{1}{2^{z}}+\frac{1}{3^{z}}+\cdots=\prod\left(1-p^{-z}\right)^{-1} \quad(\operatorname{Re} z>1),
$$

where Euler's identity on the right hand side expresses the relation of the integers to the primes. The zeros $z_{k}$ of Riemann's analytic continuation of (1) comprise the negative even integers, $-2,-4, \cdots$, and an infinite number of nontrivial zeros $z_{k}=a_{k}+i y_{k}$ in the strip $0<a_{k}<1$.

A general approach to find zeros is by continuation [1]. If $z(0)=z_{0}$ is a starting point of a path $z(\lambda)$ with tangent $\tau=z^{\prime}(\lambda)$,

$$
\tau \frac{\zeta^{\prime}(z)}{\zeta(z)}=-1
$$


then the endpoint $Z_{*}=\lim _{\lambda \rightarrow \infty} z(\lambda)$ is a zero of $\zeta(z)$, all of which are isolated. All known nontrivial zeros satisfy $\operatorname{Re} z_{k}=\frac{1}{2}$ to within numerical precision, the first three of which are $z_{1}=\frac{1}{2} \pm 14.1347 i$, $z_{2}=\frac{1}{2} \pm 21.0220 i, \quad z_{3}=\frac{1}{2} \pm 25.0109 i$. By the symmetry

$$
\zeta(s)=\zeta(1-s) \frac{\chi(1-s)}{\chi(s)}, \quad \chi(s)=\pi^{\frac{s}{2}} \Gamma\left(\frac{s}{2}\right), \quad \Gamma(z)=\int_{0}^{\infty} t^{z-1} \mathrm{e}^{-t} \mathrm{~d} t
$$

it suffices to study zeros in the half plane $\operatorname{Re}(z) \geq \frac{1}{2}$. Figure 1 illustrates root finding by (2) for the first few zeros.

Continuation (2) is determined by the prime numbers, since

$$
-\frac{\zeta^{\prime}(z)}{\zeta(z)}=-\sum \frac{\ln p}{p^{z}-1}=\sum \xi(m z), \quad \xi(z)=\sum p^{-z} \ln p \quad(\operatorname{Re} z>1),
$$

whereby

$$
\xi(z)=-\frac{\zeta^{\prime}(z)}{\zeta(z)}-\sum_{m \geq 2} \xi(m z)
$$

The poles of $\xi(z)$ at the zeros are therefore expressed by the prime number distribution.

In this paper, we study the distribution of zeros $z_{k}$ by Fourier analysis of the function

$$
\Phi(x)=x^{-\frac{1}{4}}[1-2 \sqrt{x} \varphi(x)]
$$

on $x>0$, where

$$
\varphi(x)=\sum \mathrm{e}^{-p^{2} \pi x} \log p
$$

with summation over all primes. In what follows, we put

$$
Z(\lambda)=\sum \alpha_{k} \mathrm{e}^{-\lambda\left(z_{k}-\frac{1}{2}\right)}, \quad \alpha_{k}=\gamma\left(z_{k}\right), \quad \gamma(z)=\frac{\Gamma\left(\frac{z}{2}\right)}{\pi^{\frac{z}{2}}} .
$$

The $\alpha_{k}$ are absolutely summable by Stirling's formula and the asymptotic distribution of $z_{k}$.

Theorem 1.1. In the limit as $x>0$ becomes small, we have the asymptotic behavior

$$
\Phi(x)=\frac{1}{2} \gamma\left(\frac{1}{2}\right)+Z(\ln \sqrt{x})+\frac{1}{3} \gamma\left(\frac{1}{3}\right) x^{\frac{1}{12}}+o\left(x^{\frac{1}{12}}\right) .
$$

In (9), $Z$ is evidently unbounded in the limit as $x$ approaches zero whenever a finite number of zeros $z_{k}$ exists off the critical line $\operatorname{Re} z=\frac{1}{2}$.

Corollary 1.2. If $\Phi$ is bounded, then the Riemann hypothesis is true or there are infinitely many zeros $\operatorname{Re} z_{k}>\frac{1}{2}$.

A similar relation between the distribution of $z_{k}$ and the primes is [2] [3]

$$
\frac{u-\psi_{C}(u)}{\sqrt{u}}=\sum \frac{u^{z_{k}-\frac{1}{2}}}{z_{k}}+\frac{\ln (2 \pi)+\ln \sqrt{1-u^{-2}}}{\sqrt{u}}
$$

based on the Chebyshev functions 


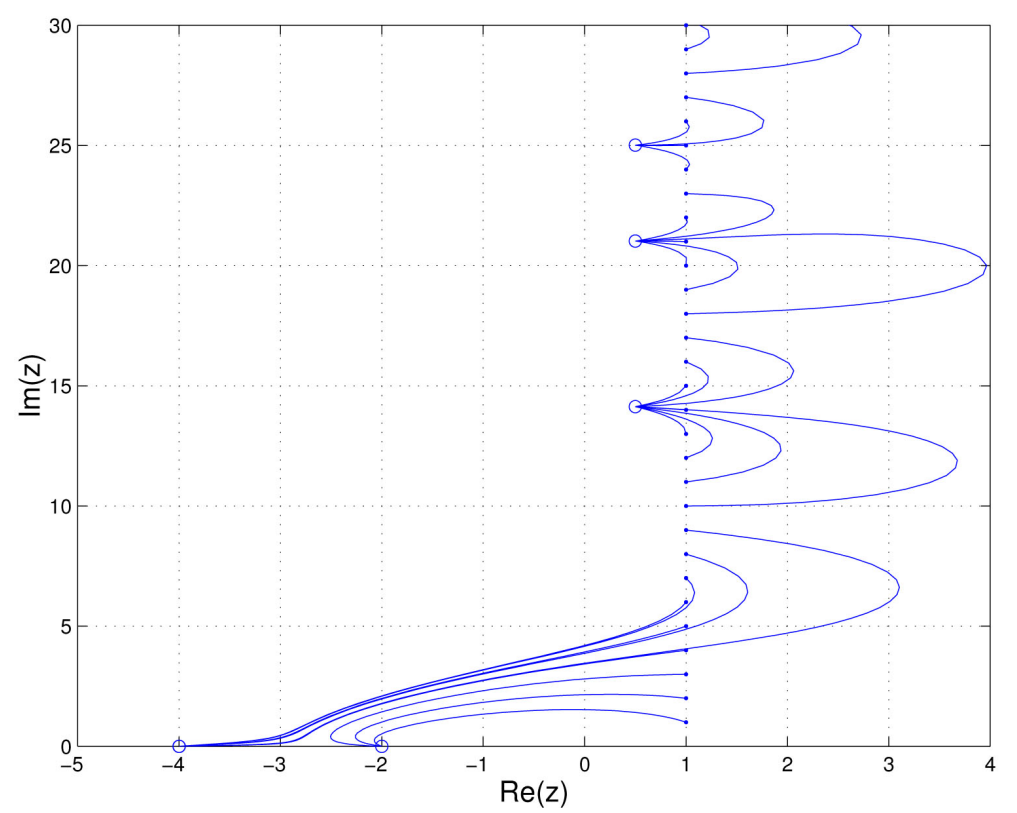

Figure 1. Shown are the trajectories of continuation $z(\lambda)$ in the complex plane $z$ by numerical integration of (2) with initial data $z_{0}=1+n i \quad(n=1,2,3, \cdots)$ indicated by small dots on $\operatorname{Re}(z)=1$. Continuation produces roots indicated by open circles, defined by finite endpoints of $z(\lambda)$ in the limit as $\lambda$ approaches infinity. The roots produced by the choice of initial data are the first three on $\operatorname{Re} z=\frac{1}{2}$ and -2 and -4 of the trivial roots.

$$
\psi_{C}(u)=\sum_{p^{k} \leq u} \ln (p), \quad \vartheta_{C}(u)=\sum_{p \leq u} \ln p,
$$

where the sum is over all primes $p$ and integers $k$. In (9), $\Phi(x)$ has a normalization by $x^{\frac{1}{4}}$ according to and $Z$ is absolutely convergent for all $x>0$, whereas in (10) $\psi_{C}(u)$ is normalized by $\sqrt{u}$ and the sum $\sum \frac{u^{z_{k}-\frac{1}{2}}}{z_{k}}$ is not absolutely convergent. Similar to Corollary 1.2, the left hand side of (10) will be bounded in the limit of large $u$ if the Riemann hypothesis is true.

Section 2 presents some preliminaries on $\zeta(z)$. Section 3 gives an integral representation of $\zeta(z)$ and a discussion on its singularity at $z=1$. In Section 4, Cauchy's integral formula is applied to derive a sum of residues associated with the $z_{k}$. The proof Theorem 1.1 follows from a Fourier transform and asymptotic analysis (Section 5). In Section 6, we illustrate a direct evaluation of $\Phi(x)$ using the primes up to one trillion, showing harmonic behavior arising from $Z$ by the first few zeros $z_{k}$. We summarize our findings in Section 7 .

\section{Background}

Our analysis begins with some known properties of $\zeta(z)$ in, e.g., [4]-[9].

Riemann obtained an analytic extension of $\zeta(z)$ by expressing $n^{-z}$ in terms of $\Gamma\left(\frac{z}{2}\right)$,

$$
\gamma(z) \zeta(z)=\int_{0}^{\infty} x^{\frac{z}{2}-1} \theta_{1}(x) \mathrm{d} x
$$

where

$$
\theta_{1}(x)=\frac{\theta(x)-1}{2}, \theta(x)=\sum_{n=-\infty}^{\infty} \mathrm{e}^{-n^{2} \pi x}
$$


Here, $\theta_{1}(x)$ satisfies $\theta_{1}(x) \sim \frac{1}{2 \sqrt{x}}$ as $x$ approaches zero by the identity $\theta\left(x^{-1}\right)=\sqrt{x} \theta(x)$ for the Jacobi function $\theta(x)^{1}$. On $\operatorname{Re} z>1$, it obtains the meromorphic expression (e.g. Borwein et al., 2006)

$$
\gamma(z) \zeta(z)=\frac{1}{z(z-1)}+f(z), \quad f(z)=\int_{1}^{\infty}\left(x^{\frac{z}{2}-1}+x^{-\frac{z}{2}-\frac{1}{2}}\right) \theta_{1}(x) \mathrm{d} x,
$$

which gives a maximal analytic continuation of $\zeta(z)$ and shows a simple pole at $z=1$ with residue 1 .

Riemann further introduced the symmetric form $Q(z) \zeta(z), Q(z)=\frac{1}{2} z(z-1) \gamma(z)$ satisfying $Q(z) \zeta(z)=Q(1-z) \zeta(1-z)$, whereby

$$
\zeta(z)=\pi^{z-1} \zeta(1-z) \frac{\Gamma\left(\frac{1}{2}-\frac{z}{2}\right)}{\Gamma\left(\frac{z}{2}\right)}=\frac{\pi^{z} \zeta(1-z)}{\cos \left(\frac{1}{2} \pi z\right) \Gamma\left(\frac{z}{2}\right) \Gamma\left(\frac{1}{2}+\frac{z}{2}\right)}=\frac{\pi^{z-\frac{1}{2}} 2^{z-1} \zeta(1-z)}{\cos \left(\frac{1}{2} \pi z\right) \Gamma(z)}
$$

using $\Gamma\left(\frac{1}{2}-\frac{z}{2}\right) \Gamma\left(\frac{1}{2}+\frac{z}{2}\right)=\frac{\pi}{\cos (\pi z)}$ and $\Gamma(z) \Gamma\left(z+\frac{1}{2}\right)=2^{1-2 z} \sqrt{\pi} \Gamma(2 z)$. Along $z=1-i y, \quad \zeta(z)$ is nonvanishing [10]-[13], allowing

$$
\frac{\zeta^{\prime}(z)}{\zeta(z)}=-\frac{\zeta^{\prime}(1-z)}{\zeta(1-z)}+\ln (2 \pi)+\frac{\pi}{2} \tan \left(\frac{\pi z}{2}\right)-\psi(z)
$$

in terms of the digamma function

$$
\psi(z)=\frac{\Gamma^{\prime}(z)}{\Gamma(z)} \sim \ln (z)+O\left(z^{-1}\right)
$$

in the limit of large $|z|$.

Lemma 2.1. In the limit of large $y$, the logarithmic derivative of $\zeta(z)$ satisfies

$$
\frac{\zeta^{\prime}(i y)}{\zeta(i y)}=-\frac{\zeta^{\prime}(1-i y)}{\zeta(1-i y)}+O(\ln y)
$$

Proof. The result follows from (17) and (16).

Lemma 2.2. Along the line $z=i y$, we have the asymptotic expansion $|\gamma(i y)| \sim \sqrt{\frac{2 \pi}{y}} \mathrm{e}^{-\frac{\pi}{2} y}$ in the limit of large $y$, whereby the $\alpha_{k}$ are absolutely summable.

Proof. Recall (8) and the asymptotic expansion $\Gamma(z)=\sqrt{2 \pi} z^{z-\frac{1}{2}} \mathrm{e}^{-z}\left[1+O\left(z^{-1}\right)\right]$ with a branch cut along the negative real axis. In the limit of large $y_{k}, y_{k} \sim \frac{2 \pi k}{\ln k}$, and hence $\left|Z_{k}\right| \sim \mathrm{e}^{-\frac{\pi^{2} k}{\ln k}}$, since $\left|\arg z_{k}\right| \sim \frac{\pi}{2}$ as $k$ becomes large. Hence, the $Z_{k}$ are absolutely summable. Numerically, their sum is small, $\sum\left|\alpha_{k}\right|=3.5 \times 10^{-5}$ based on a large number of known zeros $z_{k}$.

Lemma 2.3. In the limit of large $y$, we have

$$
\mid \gamma(\text { iy }) \frac{\zeta^{\prime}(i y)}{\zeta(i y)} \mid=O\left(y^{-\frac{1}{2}} \mathrm{e}^{-\frac{\pi}{2} y} \ln y\right) .
$$

${ }^{1}$ When $z=n$ is an integer, $\frac{\pi^{\frac{n}{2}}}{\Gamma\left(\frac{n}{2}\right)}$ is one-half the surface area of $S^{n}$. 
Proof. By Lemma 2.1-2.2, we have

$$
\left|\gamma(i y) \frac{\zeta^{\prime}(i y)}{\zeta(i y)}\right| \sim \sqrt{\frac{2 \pi}{y}}\left(\left|\frac{\zeta^{\prime}(1-i y)}{\zeta(1-i y)}\right|+O(\ln y)\right) \mathrm{e}^{-\frac{\pi}{2} y}
$$

for large $y$. Also [4] [14] [15]

$$
\left|\frac{\zeta^{\prime}(1-i y)}{\zeta(1-i y)}\right| \leq c(\ln y)^{\frac{2}{3}}(\ln \ln y)^{\frac{1}{3}}
$$

on $y>\delta$ for some positive constants $c, \delta$.

\section{An Integral Representation of $\xi(z)$}

Following the same steps leading to the Riemann integral for $\zeta(z)$, we have

$$
\gamma(z) \xi(z)=\int_{0}^{\infty} x^{\frac{z}{2}-1} \varphi(x) \mathrm{d} x=\frac{1}{z-1}+g(z),
$$

where $1 /(z-1)$ absorbs the simple pole in $\xi(z)$ at $z=1$ due to the simple pole in $\zeta(z)$ at $z=1$, leaving $g(z)$ analytic at $z=1$. Following a decomposition $g(z)=g_{2}(z)-g_{1}(z)$,

$$
g_{1}(z)=\frac{1}{2} \int_{0}^{1} x^{\frac{2 z-1}{4}} \Phi(x) \frac{\mathrm{d} x}{x}, \quad g_{2}(z)=\int_{1}^{\infty} x^{\frac{z}{2}-1} \varphi(x) \mathrm{d} x,
$$

and substitution $x=\mathrm{e}^{2 \lambda}, g(z)$ appears as the Laplace transforms

$$
g_{1}(z)=\int_{-\infty}^{0} \Phi\left(\mathrm{e}^{2 \lambda}\right) \mathrm{e}^{\lambda\left(z-\frac{1}{2}\right)} \mathrm{d} \lambda, \quad g_{2}(z)=2 \int_{0}^{\infty} \varphi\left(\mathrm{e}^{2 \lambda}\right) \mathrm{e}^{\lambda z} \mathrm{~d} \lambda .
$$

These integral expressions allow continuations to $\operatorname{Re} z>1$, respectively, the entire complex plane.

Lemma 3.1. Analytic extension of $g_{1}(z)$ extends to $z>\frac{1}{2}$.

Proof. With $z=a+i b$, the second term on the right hand side in (5) satisfies

$$
\sum_{m \geq 3}|\xi(m z)| \leq \sum_{n \geq 3} \frac{n^{-3 a} \log n}{1-n^{-a}}<-\frac{\sqrt{2} \zeta^{\prime}(3 a)}{\sqrt{2}-1},
$$

which is bounded in $\operatorname{Re} z=a>\frac{1}{2}$. Since the second term $\xi(2 z)$ in (5) is analytic in $\operatorname{Re} z=a>\frac{1}{2}$, it follows that $g(a)$ in is analytic on $a>\frac{1}{2}$. Following (5) as $a$ approaches $\frac{1}{2}$ from the right, we have

$$
\xi(a)=-\frac{1}{2 a-1}+u_{1}(a)
$$

where $u_{1}(a)$ is analytic at $a=\frac{1}{2}$. By (22), as $a$ approaches $\frac{1}{2}$ from the right, we have

$$
g_{1}(a)=-\frac{1}{2 a-1}+u_{2}(a)
$$

where $u_{2}(a)$ is analytic about $a=\frac{1}{2}$.

Figure 2 shows a numerical evaluation of $\Phi(x)$ for small $x$ evaluated for the 37.6 billion primes up to one trillion, allowing $x$ down to $2.6 \times 10^{-23} \quad(\lambda=-26)$ in view of the requirement for an accurate truncation in $\varphi(x)$ as defined by (7). The result shows asymptotic harmonic behavior in the limit as $x$ becomes small.

If the integral 

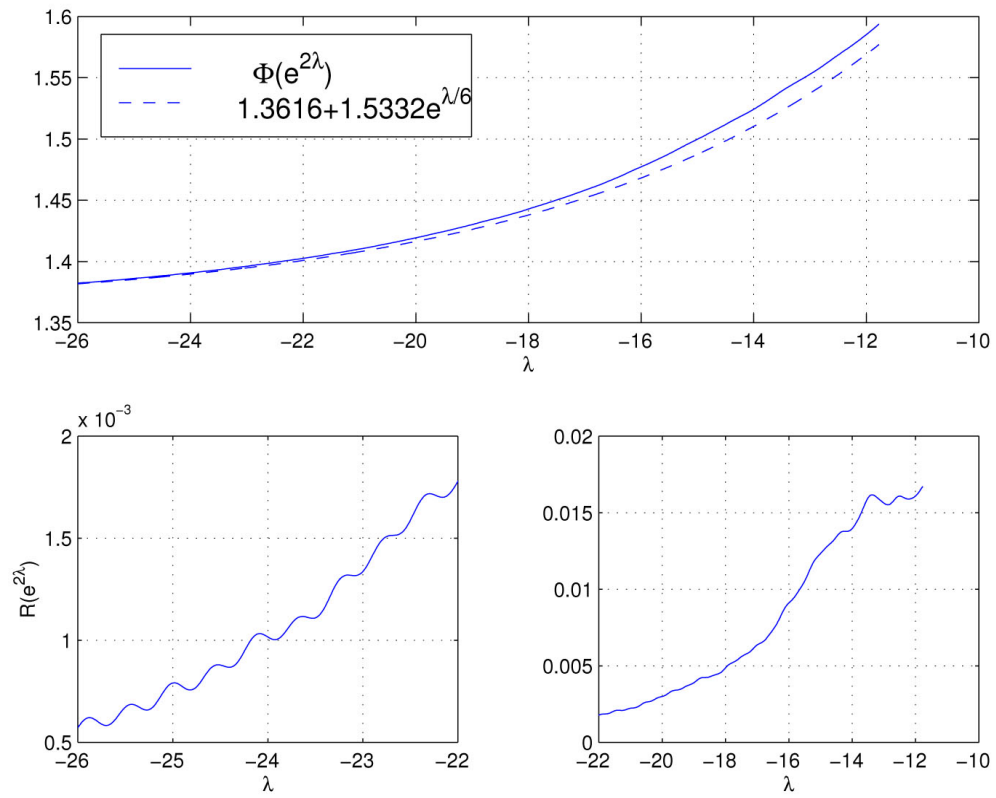

Figure 2. The top window shows $\Phi\left(\mathrm{e}^{2 \lambda}\right)$ on $\lambda \in[-26,-11.7756]$ and its leading order approximation $1.3616+1.5332 \mathrm{e}^{\frac{\lambda}{6}}$. The asymptotic harmonic behavior is apparent in the residual difference (52) between the two, shown in the bottom two windows, including the period of 2.2496 in $\lambda$ associated with the first zero $Z_{*}=\frac{1}{2} \pm 14.1347 i$.

$$
\int_{\epsilon}^{1} x^{\frac{2 z-1}{4}} \Phi(x) \frac{\mathrm{d} x}{x}
$$

is absolutely convergent as $\epsilon>0$ approaches zero, e.g., when $\Phi(x)$ is of one sign in some neighborhood of $z=0$, as in the numerical evaluation shown in Figure 2, then $g_{1}(z)$ has an analytic extension into $\operatorname{Re} z>\frac{1}{2}$ with no singularities, implying the absence of $z_{k}$ in this region. However, this requires information on the point wise behavior of $\Phi(x)$, which goes beyond the relatively weaker integrability property (23).

To make a step in this direction, we next apply a linear transform to (5) to derive the asymptotic behavior of $\Phi(x)$ in terms of the distribution $z_{k}$.

\section{A Sum of Residues Z Associated with the Non-Trivial Zeros}

Consider

$$
h(z)=\gamma(z) \frac{\zeta^{\prime}(z)}{\zeta(z)}+\frac{1}{z-1}
$$

and its Fourier transform

$$
H(\lambda)=\int_{a-i \infty}^{a+i \infty} h(z) \mathrm{e}^{-\lambda z} \frac{\mathrm{d} z}{2 \pi i} .
$$

Lemma 4.1. $h(z)$ has a simple pole at $z=1$ with residue 1 and simple poles at each of the nontrivial zeros $z_{k}$ of $\zeta(z)$ with residue $Z_{k}$.

Proof. We have (e.g. Borwein et al. 2006)

$$
\zeta_{1}(z)=\frac{1}{2} z(z-1) \gamma(z) \zeta(z), \quad \frac{\zeta_{1}^{\prime}(z)}{\zeta_{1}(z)}=B+\sum_{k}\left(\frac{1}{z-z_{k}}+\frac{1}{z_{k}}\right),
$$

where $B$ is a constant, so that 


$$
\gamma(z) \frac{\zeta^{\prime}(z)}{\zeta(z)}+\frac{1}{z-1}=\gamma(z)\left[B+\Sigma_{k}\left(\frac{1}{z-z_{k}}+\frac{1}{z_{k}}\right)\right]+A(z)
$$

Here

$$
A(z)=\frac{1-\gamma(z)}{z-1}-\frac{\gamma(z)}{z}-2 \frac{\gamma(z) \psi(z)}{\ln \pi},
$$

where $\psi(z)$ denotes the digamma function as before, includes contributions from the logarithmic derivative of the factor to $\zeta(z)$ in (31), whose singularities are restricted to the trivial zeros of $\zeta(z)$.

We now consider the Fourier integral over $\operatorname{Re} z=a$ as part of contour integration closed over $z=x \pm i Y$ and $\operatorname{Re} z=0$.

Proposition 4.2. The Fourier transform of $h(z)$ over $\operatorname{Re} z>\sup a_{k}$ satisfies

$$
H(\lambda)=\mathrm{e}^{-\frac{\lambda}{2}} Z(\lambda)+O(1)
$$

in the limit of large $\lambda<0$.

Proof. Integration over $z=x+i Y \quad(0<x<a)$ gives

$$
\mathrm{e}^{-i Y} \int_{i Y}^{i Y+a} h(z) \mathrm{e}^{-\lambda x} \frac{\mathrm{d} x}{2 \pi i}=\mathrm{e}^{-i Y} \int_{i Y}^{i Y+a} \frac{\Gamma\left(\frac{z}{2}\right)}{\pi^{\frac{z}{2}}} \frac{\zeta^{\prime}(z)}{\zeta(z)} \frac{\mathrm{d} x}{2 \pi i}+O\left(Y^{-1}\right),
$$

where we choose $Y$ to be between two consecutive values of $y_{k}$. We have

$$
\int_{i Y}^{i Y+a} \frac{\Gamma\left(\frac{z}{2}\right)}{\pi^{\frac{z}{2}}} \frac{\zeta^{\prime}(z)}{\zeta(z)} \frac{\mathrm{d} x}{2 \pi i} \sim \gamma_{k} \int_{i Y}^{i Y+a} \frac{\zeta^{\prime}(z)}{\zeta(z)} \frac{\mathrm{d} x}{2 \pi i} \sim \frac{\gamma_{k}}{2 \pi i} \ln (2 a-1)\left[1+\frac{4 a i\left(y_{k}-Y\right)}{1-2 a}+\pi i\right] .
$$

In the limit as $k$ approaches infinity, $y_{k}-Y$ approaches zero and $\left|\gamma_{k}\right|$ becomes small by Lemma 2.2., whence

$$
\left(\int_{i Y}^{i Y+a}-\int_{-i Y}^{-i Y+a}\right) \frac{\Gamma\left(\frac{z}{2}\right)}{\pi^{\frac{z}{2}}} \frac{\zeta^{\prime}(z)}{\zeta(z)} \frac{\mathrm{d} x}{2 \pi i} \sim \ln (2 a-1) \operatorname{Im} \gamma_{k}=O\left(\ln (2 a-1) \sqrt{\frac{2 \pi}{y_{k}}} \mathrm{e}^{-\frac{\pi}{2} y_{k}}\right) .
$$

Next, integration over $z=i y$ with a small semicircle around $z=0$ obtains an $O(1)$ result in the limit of large $\lambda$ by application of Lemma 2.1-2.3 and the Riemann-Lebesgue Lemma. The result now follows in the limit as $k$ approaches infinity, taking into account the residue sum $\mathrm{e}^{-\overline{2}} Z(\lambda)$ associated with the $z_{k}$ and absolute summability of the $\alpha_{k}$.

\section{Proof of Theorem 1.1}

Multiplying (5) by $\gamma(z)$, we have

$$
\gamma(z) \xi(z)=-\gamma(z) \frac{\zeta^{\prime}(z)}{\zeta(z)}-\gamma(z) \sum_{m \geq 2} \xi(m z)
$$

that is, by (22) and (29),

$$
\frac{1}{z-1}+g(z)=-h(z)+\frac{1}{z-1}-\gamma(z) \sum_{m \geq 2} \xi(m z)
$$

We thus consider

$$
g_{1}(z)=g_{2}(z)+h(z)+\gamma(z) \sum_{m \geq 2} \xi(m z)
$$

which $a b$ initio is defined on $\operatorname{Re} z>1$ by Euler's identity with Fourier transform 


$$
G_{1}(\lambda)=\int_{a-i \infty}^{a+i \infty} g_{1}(z) \mathrm{e}^{-\lambda z} \frac{\mathrm{d} z}{2 \pi i}=\mathrm{e}^{-\frac{\lambda}{2}} \Phi\left(\mathrm{e}^{2 \lambda}\right) .
$$

Turning to the right hand side of (40), we consider the coefficients

$$
c_{m}(z)=\frac{\gamma(z)}{\gamma(m z)}, C_{m}=\frac{1}{m} \gamma\left(\frac{1}{m}\right)(m \geq 1)
$$

Here, $C_{m}=m^{-1} \gamma(1 / m)$ since $\gamma(1)=1$. In particular, $C_{2}=\frac{1}{2} \gamma\left(\frac{1}{2}\right)$ and

$m^{-1} c_{m}\left(m^{-1} z\right)=1+\left(\frac{1}{2} \ln \pi+\frac{1}{2} \gamma\right) z+O\left(z^{2}\right)$ has a well defined limit and $C_{m} \rightarrow 2$ in the limit as $m$ becomes arbitrarily large.

Lemma 5.1. The sum $\sum_{m \geq n} \xi(m z)$ is well-defined on $\operatorname{Re} z>\frac{1}{n}$.

Proof. The result follows from the case $n=2$. By the Prime Number Theorem, $p_{k} \sim k \ln k$, whereby summation over the tails $k \geq n$ satisfy

$$
\sum_{k \geq n}^{\infty} \frac{\ln \left(p_{k}\right)}{p_{k}^{2 a}} \sim \sum_{k \geq n}^{\infty}\left[\frac{1}{k^{2 a} \ln (k)^{2 a-1}}+\frac{\ln \ln (k)}{(k \ln (k))^{2 a}}\right]<\infty
$$

whenever $a>\frac{1}{2}$. Hence, for $z=a+i y,\left|\sum p^{-2 z} \ln p\right| \leq \sum p^{-2 a} \ln p<\infty$ whenever $a>\frac{1}{2}$. It follows that

$$
\left|\sum_{m \geq 2} \xi(m z)\right| \leq \sum_{m \geq 2} \sum_{p} p^{-(m-2) a} p^{-2 a} \ln p \leq \sum_{m \geq 0} 2^{-m} \sum_{p} p^{-2 a} \ln p=\sum_{p} p^{-2 a} \ln p<\infty
$$

on $\operatorname{Re} z>\frac{1}{2}$.

Lemma 5.2. For any $m \geq 2$, the Fourier transform of $\frac{c_{m}(z)}{m z-1}$ over $\operatorname{Re} z=a>\frac{1}{2}$ satisfies

$$
D_{m}(\lambda)=C_{m} \mathrm{e}^{-\frac{\lambda}{m}}+o(1)
$$

Proof. The Fourier integral can be obtained in a contour integration with closure over $z=i y$ and the edges $z=x+i Y \quad(0<x<a)$ for large $\pm Y$. In the notation (42), it obtains a residue $C_{m}=m^{-1} c_{m}(1 / m)=m^{-1} \gamma(1 / m)$ at $z=1 / m$, since $\gamma(1)=1$, whence

$$
D_{m}(\lambda)=C_{m} \mathrm{e}^{-\frac{\lambda}{m}}+\mathrm{e}^{\frac{\lambda}{2}} \int_{-\infty}^{\infty} \frac{C_{m}(i y)}{i m y-1} \mathrm{e}^{-i \lambda y} \frac{\mathrm{d} y}{2 \pi} .
$$

The integral (46) exists by virtue of a removable singularity of $c_{m}(z)$ at $z=0$. It asymptotically decays to zero for large $\lambda$ when $m \geq 2$ by the Riemann-Lebesgue Lemma.

We now consider (40) with (22),

$$
g_{1}(z)=g_{2}(z)+h(z)+\sum_{m \geq 2}\left(\frac{c_{m}(z)}{m z-1}+c_{m}(z) g(m z)\right)=h(z)+\sum_{m=2}^{N} \frac{c_{m}(z)}{m z-1}+r_{N}(z)
$$

with a remainder

$$
r_{N}(z)=g_{2}(z)+\sum_{m \geq 2} c_{m}(z) g(m z)+\gamma(z) \sum_{m \geq N+1} \xi(m z) .
$$

Lemma 5.3. For $N \geq 3$, the Fourier transform

$$
\mathrm{e}^{\frac{\lambda}{2}} R_{N}(\lambda)=\int_{a-i \infty}^{a+i \infty} r_{N}(z) \mathrm{e}^{-\lambda\left(z-\frac{1}{2}\right)} \frac{\mathrm{d} z}{2 \pi i}=o(1)
$$


in the limit of large $\lambda<0$.

Proof. Since $r_{N}(z)$ is analytic in $\operatorname{Re} z>\frac{1}{3}$, we are at liberty to consider the transform $\mathrm{e}^{\frac{\lambda}{2}} R_{N}(\lambda)$ on $a=1 / 2$. The result follows from the Riemann-Lebesgue Lemma.

Proof of Theorem 1.1. The Fourier transform of (47) is

$$
G_{1}(\lambda)=H(\lambda)+D_{2}(\lambda)+D_{3}(\lambda)+R_{3}(\lambda) .
$$

By Proposition 4.2 and Lemmas 5.1-5.2, we have

$$
\mathrm{e}^{-\frac{\lambda}{2}} \Phi\left(\mathrm{e}^{2 \lambda}\right)=\mathrm{e}^{-\frac{\lambda}{2}} Z(\lambda)+C_{2} \mathrm{e}^{-\frac{\lambda}{2}}+C_{3} \mathrm{e}^{-\frac{\lambda}{3}}+o\left(\mathrm{e}^{-\frac{\lambda}{2}}\right) .
$$

With $x=\mathrm{e}^{2 \lambda}$, Theorem 1.1 now follows.

\section{Numerical Illustration of Asymptotic Harmonic Behavior}

The harmonic behavior emerges in

$$
R(x)=-\Phi(x)-C_{2}-C_{3} x^{\frac{1}{12}} .
$$

To search for higher harmonics $Z_{i}(\lambda)$ associated with the zeros $z_{i}$ in $\lambda \in[-26,-11.7759]=\left[\lambda_{1}-\lambda_{2}, \lambda_{1}+\lambda_{2}\right]$, we compare the spectrum of $\Phi\left(\mathrm{e}^{2 \lambda}\right)$ by taking a Fast Fourier Transform with respect to $\alpha$,

$$
\lambda(\alpha)=\lambda_{1}+\lambda_{2} \cos \alpha \quad(\alpha \in[0,2 \pi]),
$$

and compare the results with an analytic expression for the Fourier coefficients of the $Z_{i}(\lambda) \quad(i=1,2, \cdots)$,

$$
c_{n i}\left[\lambda_{1}, \lambda_{2}\right]=2 \operatorname{Re}\left\{(-i)^{n} \gamma_{i} \mathrm{e}^{-i \lambda_{1} z_{i}} J_{n}\left(-\lambda_{2} z_{i}\right)\right\},
$$

where $J_{n}(z)$ denotes the Bessel function of the first of order $n$. Figure 3 shows the first 21 harmonics in our evaluation of $\Phi(x)$, which is about the maximum that can be calculated by direct summation in quad precision.

\section{Conclusions}

The zeros $z_{k}=a_{k}+i y_{k}$ of the Riemann-zeta function are endpoints of continuation, defined by an expressed by a regularized sum $\Phi(x)$ over the prime numbers defined by (6).

The zeros $z_{k}$ of $\zeta(z)$ introduce asymptotic harmonic behavior in $\Phi\left(\mathrm{e}^{2 \lambda}\right)$ as a function of $\lambda<0$, defined by the sum $Z(\lambda)$ of residues of the $z_{k}$, shown in Figure 2, Figure 3. Primes up to 4 billion are needed to identify the first 4 harmonics, up to 70 billion for the 10 and up to 1 trillion for the first 21. It appears that, effectively, the prime number range scales exponentially with the number of harmonics it contains.

Theorem 1.1 describes a correlation between the distribution of the primes and the distribution of the nontrivial zeros $z_{k}$. Suppose there are a finite number of zeros $z_{k}$ in $\operatorname{Re} z>\frac{1}{2}$. We may then consider $k^{*}$ for which $a_{k^{*}}=\max a_{k}$ gives rise to dominant exponential growth in $Z(\lambda)$ in the limit as $\lambda<0$ becomes large. This observation leads to Corollary 1.2. $Z$ can remain bounded in $x>0$ only if the Riemann hypothesis is true, or if $Z(\lambda)$ remains fortuitously bounded as an infinite sum over $a_{k}>\frac{1}{2}$ with no maximum in $a<1$. Conversely, Riemann hypothesis implies

$$
\lim _{x \rightarrow 0^{+}} \Phi(x)=\frac{1}{2} \gamma\left(\frac{1}{2}\right) \simeq 1.3616
$$

According to (9) and our numerical calculation shown in Figure 3, the zeros $z_{k}$ explored to large $k$ by 


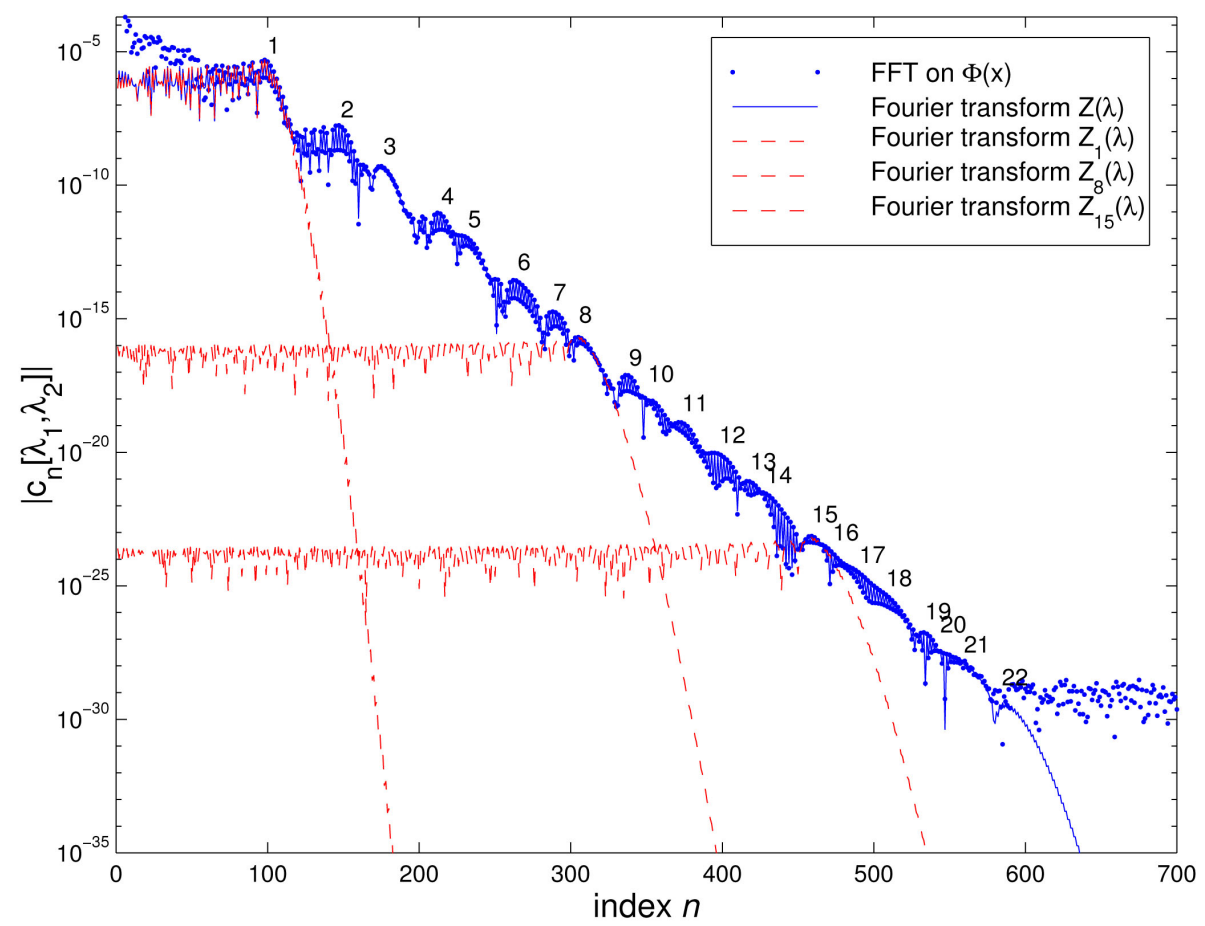

Figure 3. Shown are the absolute values of the Fourier coefficients $c_{n}\left[\lambda_{1}, \lambda_{2}\right]$ of $\Phi\left(\mathrm{e}^{2 \lambda}\right)$ obtained by a Fast Fourier Transform (FFT) of (52) on the computational domain (53), where $\lambda_{1}=-26, \lambda_{2}=-11.7756$ covers 32 periods of $Z_{1}(\lambda)$ (dots), on the basis of the $37,607,912,2019$ primes up to $1,000,000,000,0039$. The resulting spectrum is compared with the exact spectra $C_{n i}\left[\lambda_{1}, \lambda_{2}\right]$ of the $Z_{i}(\lambda)$ given by the analytic expression (54) for $i=1,2,3, \cdots$ (continuous line). Shown are also the individual spectra of $Z_{i}(\lambda)$ for $i=1,8$ and 15 associated with the zeros $Z_{1}, Z_{8}$ and $z_{15}$. The match between the computed and exact spectra accurately identifies the first 21 harmonics of $Z(\lambda)$ in $\Phi$ out of 22 shown, corresponding to the first 21 nontrivial zeros $z_{i}$ of $\zeta(z)$.

existing numerical experiments effectively probe (and constrain) a distribution in primes which extends exponentially large in $k$.

\section{Acknowledgements}

The author gratefully acknowledges stimulating discussions with Fabian Ziltener and Anton F.P. van Putten. Some of the manuscript was prepared at the Korea Institute for Advanced Study, Dongdaemun-Gu, Seoul. This research was supported in part by the National Science Foundation through TeraGrid resources provided by Purdue University under grant number TG-DMS100033. We specifically acknowledge the assistance of Vicki Halberstadt, Rich Raymond and Kimberly Dillman. The computations have been carried out using Lahey Fortran 95.

\section{References}

[1] Keller, H.B. (1987) Numerical Methods in Bifurcation Problems. Springer Verlag/Tata Institute for Fundamental Research, Berlin.

[2] Hadamard, J. (1893) Etude sur les propriétés des fonctions entières et en particulier d'une fonction. Journal de Mathématiques Pures et Appliquées, 9, 171-216.

[3] von Mangoldt, H. (1985) Zu Riemann’s Abhandlung “Über die Anzahl der Priemzahlen unter einer gegebenen Grösse”. Journal für die Reine und Angewandte Mathematik, 114, 255-305.

[4] Titchmarsh, E.C. (1986) The Theory of the Riemann Zeta-Function. 2nd Edition, Oxford. 
[5] Lehmer, D.H. (1988) The Sum of Like Powers of the Zeros of the Riemann Zeta Function. Mathematics of Computation, 50, 265-273. http://dx.doi.org/10.1090/S0025-5718-1988-0917834-X

[6] Dusart, P. (1999) Inégalités explicites pour $\psi(X), \theta(X), \pi(X)$ et les nombres premiers. Comptes Rendus Mathematiques (Mathematical Reports) des l'Academie des Sciences, 21, 53-59.

[7] Keiper, J.B. (1992) Power Series Expansions of Riemann's $\xi$ Function. Mathematics of Computation, 58, 765-773.

[8] Ford, K. (2002) Zero-Free Regions for the Riemann Zeta Function. Number Theory for the Millenium, 2, 25-26.

[9] Borwein, P., Choi, S., Rooney, B. and Weirathmueller, A. (2006) The Riemann Hypothesis. Springer Verlag, Berlin.

[10] Littlewood, J.E. (1922) Researches in the Theory of the Riemann $\xi$-Function. Proceedings of the London Mathematical Society, Series 2, 20, 22-27.

[11] Littlewood, J.E. (1926) On the Riemann Zeta-Function. Proceedings of the London Mathematical Society, Series 2, 24, 175-201. http://dx.doi.org/10.1112/plms/s2-24.1.175

[12] Littlewood, J.E. (1928) Mathematical Notes (5): On the Function $1 / \zeta(1+t i)$. Proceedings of the London Mathematical Society, Series 2, 27, 349-357. http://dx.doi.org/10.1112/plms/s2-27.1.349

[13] Wintner, A. (1941) On the Asymptotic Behavior of the Riemann Zeta-Function on the Line $\sigma=1$. American Journal of Mathematics, 63, 575-580. http://dx.doi.org/10.2307/2371370

[14] Richert, H.E. (1967) Zur Abschätzung der Riemannschen Zetafunktion in der Nähe der Vertikalen $\sigma=1$. Mathematische Annalen, 169, 97-101. http://dx.doi.org/10.1007/BF01399533

[15] Cheng, Y. (1999) An Explicit Upper Bound for the Riemann Zeta Function near the Line $\sigma=1$. Rocky Mountain Journal of Mathematics, 29, 115-140. http://dx.doi.org/10.1216/rmjm/1181071682 
Scientific Research Publishing (SCIRP) is one of the largest Open Access journal publishers. It is currently publishing more than 200 open access, online, peer-reviewed journals covering a wide range of academic disciplines. SCIRP serves the worldwide academic communities and contributes to the progress and application of science with its publication.

Other selected journals from SCIRP are listed as below. Submit your manuscript to us via either submit@scirp.org or Online Submission Portal.
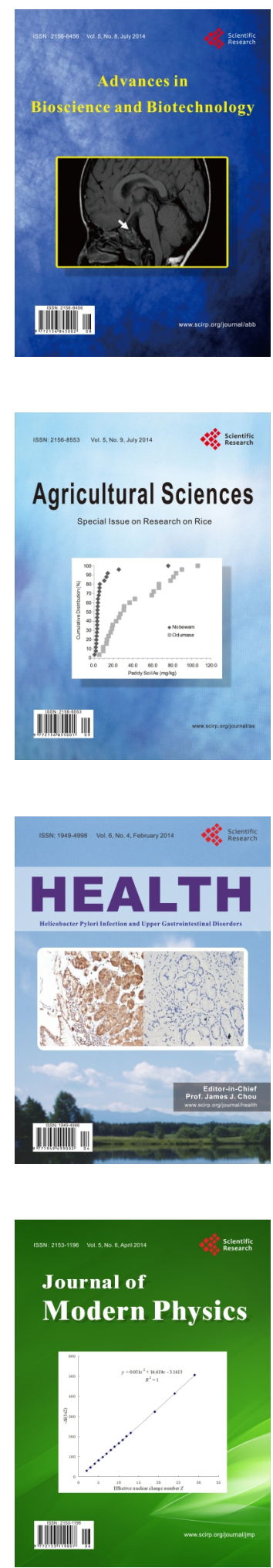
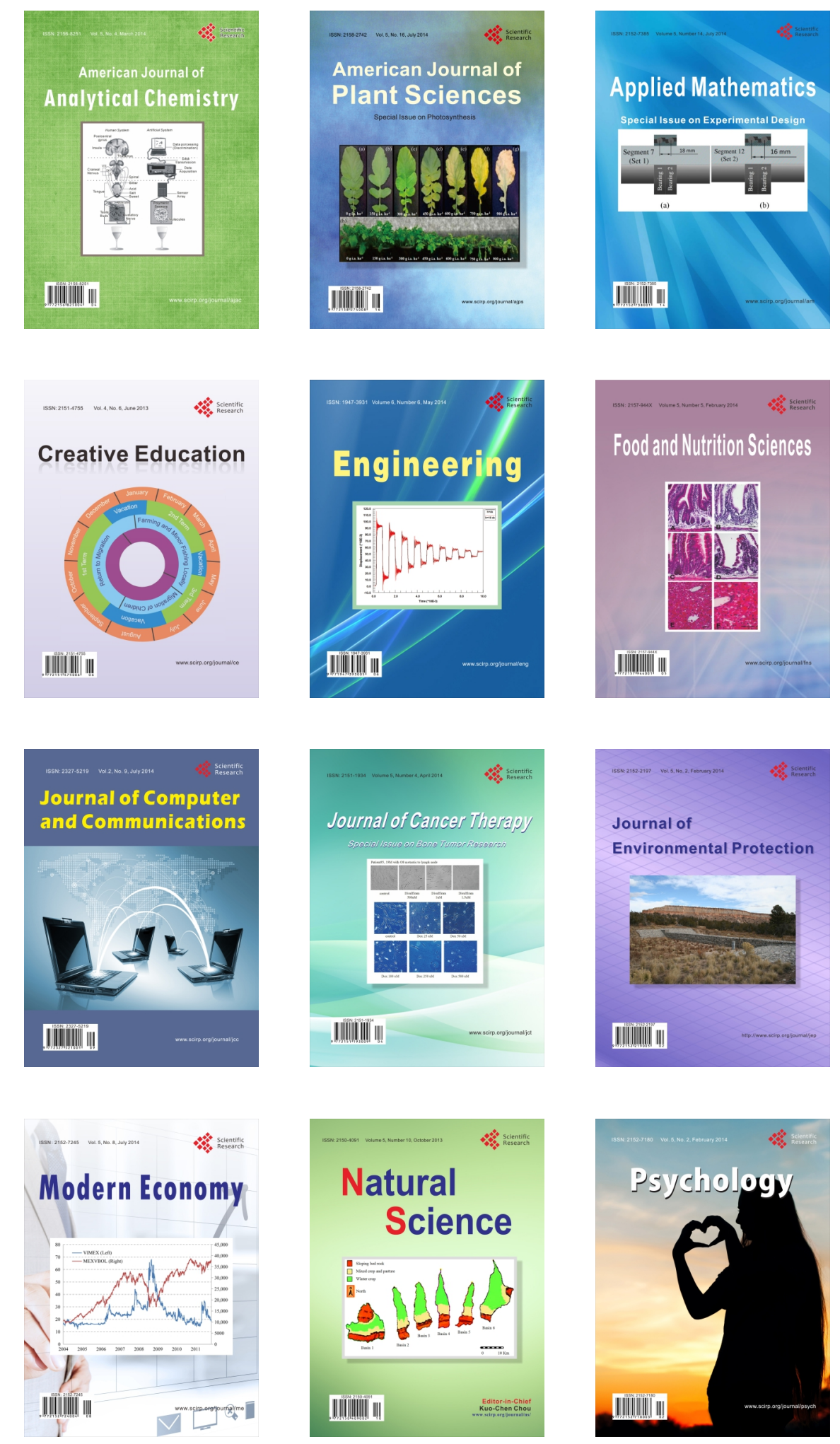